\title{
Distributed Human-Machine Systems: Progress and Prospects
}

\author{
Jeffrey M. Bradshaw \\ Florida Institute for Human and Machine Cognition, USA \\ jbradshaw@ihmc.us
}

Advances in neurophysiological and cognitive science research have fueled a surge of research aimed at more effectively combining human and machine capabilities. In this talk we will give and overview of progress and prospects for four current thrusts of technology development resulting from this research: brain-machine interfaces, robotic prostheses and orthotics, cognitive and sensory prostheses, and software and robotic assistants. Following the overview, we will highlight the unprecedented social ethics issues that arise in the design and deployment of such technologies, and how they might be responsibly considered and addressed. 